\title{
Dielectron production in proton-nucleus reactions
}

\author{
L. Xiong, J. Q. Wu, Z. G. Wu, C. M. Ko, and J. H. Shi \\ Cyclotron Institute, Center for Theoretical Physics and Physics Department, \\ Texas A\&M University, College Station, Texas 77843
}

(Received 15 August 1989)

\begin{abstract}
Dielectron production in proton-nucleus reactions is studied in the cascade model. In addition to production from the proton-neutron bremsstrahlung and the decay of delta, we have also taken into account the contribution from the pion-nucleon interaction. The calculated dielectron invariant mass spectra are compared to the data obtained recently for proton-Be reactions at incident energies of $1.05,2.1$, and $4.9 \mathrm{GeV}$.
\end{abstract}

Experiments ${ }^{1,2}$ were carried out recently at the Bevalac to measure dielectrons from the reaction of proton and heavy ions with the nuclear target. These experiments are very useful for probing the property of hot dense nuclear matter which is expected to be created in the initial stage of heavy-ion collisions. ${ }^{3-5}$ Although such a hot dense nuclear matter is unlikely to be produced in proton-nucleus reactions, the study of dielectron production from these reactions is essential for understanding the relative importance of the various processes which contribute to its production. Theoretical work up to now has been concentrated on dielectron production from the proton-neutron bremsstrahlung, ${ }^{6,7}$ from the decay of delta, ${ }^{8}$ and from the pion-pion annihilation. ${ }^{3-5,9}$ The annihilation of the secondary pions produced in the interaction on nucleons can also produce dielectrons and has been overlooked so far. In this paper, we shall include this contribution to dielectron production in proton-nucleus reactions.

To describe the reaction of a proton with the nuclear target, we use the cascade model ${ }^{10}$ to take into account the multiple collisions of the incident proton with the target nucleons, the production and decay of deltas, and also the interaction of pions, which result from delta decays, with nucleons. The cascade model should be reasonable for reactions at incident energies below about $2 \mathrm{GeV}$. For higher energies, multipion production ${ }^{9}$ in nucleon-nucleon interaction becomes important and the normal cascade model should be modified to include such a mechanism. This has not been done in the present work. The contribution from two-pion annihilation is thus negligible and will not be included. The dielectron production is then determined perturbatively as in photon ${ }^{11}$ and kaon ${ }^{12}$ production. Its production probability at each impact parameter is calculated by adding contributions from the protonneutron bremsstrahlung, the delta decay, and the pionnucleon interaction. The total dielectron production cross section is then obtained by integrating the production probability over the impact parameter.

The cross section for dielectron production from proton-neutron interaction has been evaluated microscopically in Refs. 6 and 7, and in the soft-photon approximation with the phase-space correction in Ref. 8. For simplicity, we shall use in the present work the soft-photon approximation with the phase-space correction. In this approximation, the cross section for producing a dielectron pair of invariant mass $M$ and momentum $\mathbf{p}$ is given by

$$
\frac{d \sigma_{n p}^{e^{+} e^{-}}}{d^{3} \mathrm{p} d M} \approx \frac{a^{2}}{3 \pi^{3}} \frac{\bar{\sigma}(s)}{M E^{3}} \frac{R_{2}\left(\sqrt{s_{2}}\right)}{R_{2}(\sqrt{s})} .
$$

In the above, $\alpha$ is the fine-structure constant and $\bar{\sigma}(s)$ is the momentum-transfer weighted proton-neutron elastic cross section at a center-of-mass energy $\sqrt{s}$. ${ }^{9}$ The function $R_{2}(\sqrt{s})$ is the Lorentz invariant two-body phasespace integral of the final two nucleons of energy $s$. The energy of the two nucleons after emitting a dielectron with energy $E$ is denoted by $s_{2}$. In Ref. 7, the soft-photon approximation with the phase-space correction gives a smaller contribution than that of the explicit diagrammatical calculations. But the approximation of using only the pion exchange in Ref. 7 may not be adequate. In Ref. 6, comparisons have been made between the soft-photon approximation without the phase-space correction with the explicit evaluation of the bremsstrahlung diagrams including the exchange of other mesons besides the pion. Although the former is seen to lead to a larger contribution than that from the latter, the inclusion of the phasespace correction, however, improves the agreement between them.

Deltas are produced in the cascade model from nucleon-nucleon inelastic scatterings with their masses being determined according to the following probability distribution $^{13}$

$$
P\left(m_{\Delta}\right)=\frac{\frac{1}{4} \Gamma^{2}(q)}{\left(m_{\Delta}-m_{0}\right)^{2}+\frac{1}{4} \Gamma^{2}(q)} .
$$

In the above, the centroid is $m_{0}=1232 \mathrm{MeV}$ and the width $\Gamma(q)$ is given by

$$
\Gamma(q)=\frac{0.47 q^{3}}{\left[1+0.6\left(q / m_{\pi}\right)^{2}\right] m_{\pi}^{2}},
$$

where $m_{\pi}$ is the pion mass and $q$ is its momentum in the $\Delta$ rest frame. According to Ref. 13, the use of a momentum dependent width of Eq. (2) instead of the constant width of $115 \mathrm{MeV}$ in the original cascade model of Cugnon et al. ${ }^{10}$ leads to a reduced pion yield in heavy-ion collisions and is considered to be an improved modeling of delta production.

The partial width of delta decaying into the dielectron 
with invariant mass $M$ can be evaluated according to the diagram in Fig. 1. In our calculation, the $\gamma N \Delta$ transition vertex is taken from Ref. 14 and contains contributions from magnetic dipole, electric quadrupole, and Coulomb quadrupole interactions. Keeping only the dominated magnetic dipole term, we have evaluated analytically the diagram in Fig. 1. Details of the calculation will be published elsewhere. ${ }^{15}$ The magnetic dipole form factor at the $\gamma N \Delta$ vertex has been extracted in Ref. 14 for spacelike photon and has a value of $\approx 3$ for real photon. For timelike photon as in the delta decay into the dielectron pair, no experimental information is known. In Ref. 8, the form factor is taken to be the same as that for pion-pion annihilation into the dielectron and is dominated by the rho meson. Since no such form factor has been used in the proton-neutron bremsstrahlung, ${ }^{6,7}$ we do not see the reason for using it in the delta decay. To account for the structure of the vertex, we introduce instead the following form factor, $\Lambda_{1}^{2} /\left(\Lambda_{1}^{2}+q^{2}\right)$, where $q$ is the photon three momentum. To reproduce the experimental branching ratio of $0.6 \%$ for a delta of mass $m_{\Delta}=1232 \mathrm{MeV}$ decaying into a photon requires a cutoff parameter $\Lambda_{1} \approx 0.725$ $\mathrm{GeV}$. The probability that a delta decays into a dielectron pair with invariant mass $M$ is thus given by the ratio of this partial width to the delta total width which is essentially given by Eq. (3). Our treatment of the delta contribution to dielectron production is different from that of Ref. 8 in which not only a different form factor but also the soft-photon approximation has been used. Leaving out the form factor, we find that for a delta of given mass the dielectron yield obtained with the soft-photon approximation is larger than that from the exact calculations. The difference is about $50 \%$ at small dielectron invariant masses but increases to an order of magnitude at larger invariant masses.

In the cascade model, pions are created from the decay of deltas. Although the interaction of a pion with the nucleon is most likely to form a delta again, it is possible that the total center-of-mass energy of the pion and the nucleon is such that the delta formation cross section is negligible. In this case, we need to take into account the contribution to dielectron production from the pionnucleon interaction. Energetically, we expect that dielectron production from the pion-nucleon bremsstrahlung is less important than that from pion annihilation on nucleon and is thus neglected in the present work. The contribu-

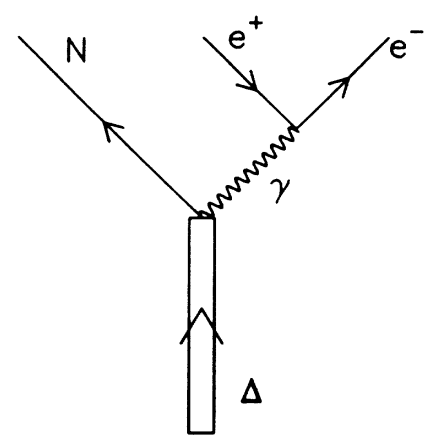

FIG. 1. Feynman diagram for delta decay into the dielectron. tion from pion annihilation on nucleon is calculated in the present work. The contribution from pion annihilation on nucleon is calculated according to the diagrams in Fig. 2. Diagram (d), which contains a four particle vertex, is a result of the gauge invariance as we used the pseudovector coupling for the pion-nucleon interaction. In diagrams (b) and (c), we do not allow delta in the intermediate state as it is treated explicitly in the cascade model and its contribution to dielectron production has already been included. These diagrams are evaluated numerically. Details of this calculation will also be published in Ref. 15 . To take into account the complicated structure of the strong interaction vertex and also to preserve the gauge invariance, an overall form factor of the dipole type is introduced, i.e., $F\left(q^{2}\right)=\Lambda_{2}^{2} /\left(\Lambda_{2}^{2}+q^{2}\right)$, where $q$ is the three-momentum transfer of the nucleon and $\Lambda_{2}$ is the cutoff parameter. Such a form factor with $\Lambda_{2} \approx 0.6 \mathrm{GeV}$ has been successfully used in studying the photopion production, ${ }^{16}$ which is a similar process as the dielectron production. We have also included the pion electromagnetic form factor of the form used in Ref. 3 at the $\gamma \pi \pi$ vertex of diagram (a). Another way of preserving the gauge invariance in the presence of the strong interaction form factor is to introduce additional diagrams in which the virtual photon is emitted from a heavy meson of mass $\Lambda_{2}$ as in Ref. 7. As can be shown easily, the latter method is, however, not applicable to the present case as the additional diagrams will simply cancel the effect from the strong interaction form factor.

The calculated total dielectron invariant mass spectra are shown in Figs. 3-5 by the solid curves for the reaction of a proton with the Be target at three incident energies of $1.05,2.1$, and $4.9 \mathrm{GeV}$. In order to compare to the experimental data ${ }^{1,2}$ shown by the solid squares, the theoretical results have been corrected for the detector acceptance filter which requires that we also determine the momentum distribution of the dielectron pairs. This reduces substantially the dielectron yield at small invariant masses. The theoretical results are seen to have the right magni-

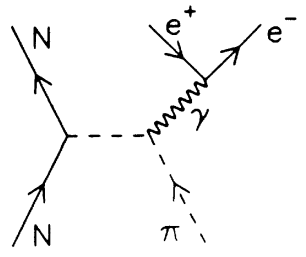

(a)

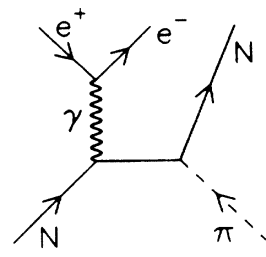

(c)

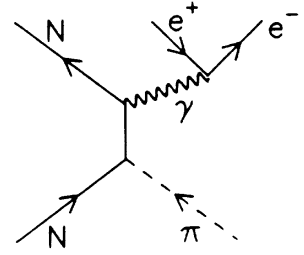

(b)

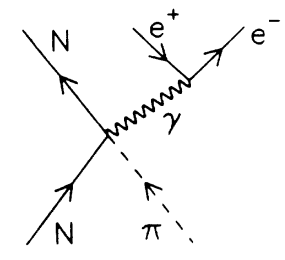

(d)
FIG. 2. Feynman diagrams for dielectron production from pion annihilation on the nucleon. 


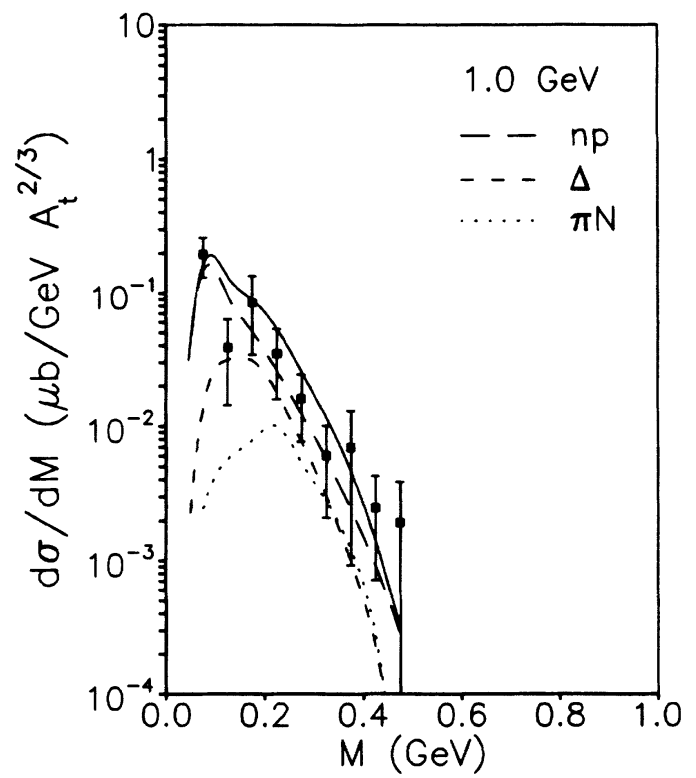

FIG. 3. Dielectron invariant mass spectra for $p+$ Be reaction at $1.05 \mathrm{GeV}$. The experimental data of Refs. 1 and 2 are given by the solid squares, while the theoretical total yield is given by the solid curve. The contributions from the proton-neutron bremsstrahlung, the delta decay, and the pion-nucleon interaction are given by the long-dashed, short-dashed, and dotted curves, respectively.

tude. To understand the results more closely, we have also shown in Figs. 3-5 the contributions to dielectron production from the three different processes. The contribution from the proton-neutron bremsstrahlung is given by the long-dashed curves and is the dominant one at all three energies. The contribution from the delta decay is denoted by the short-dashed curves and is seen to be unimportant. The dotted curves are from the pion-nucleon in-

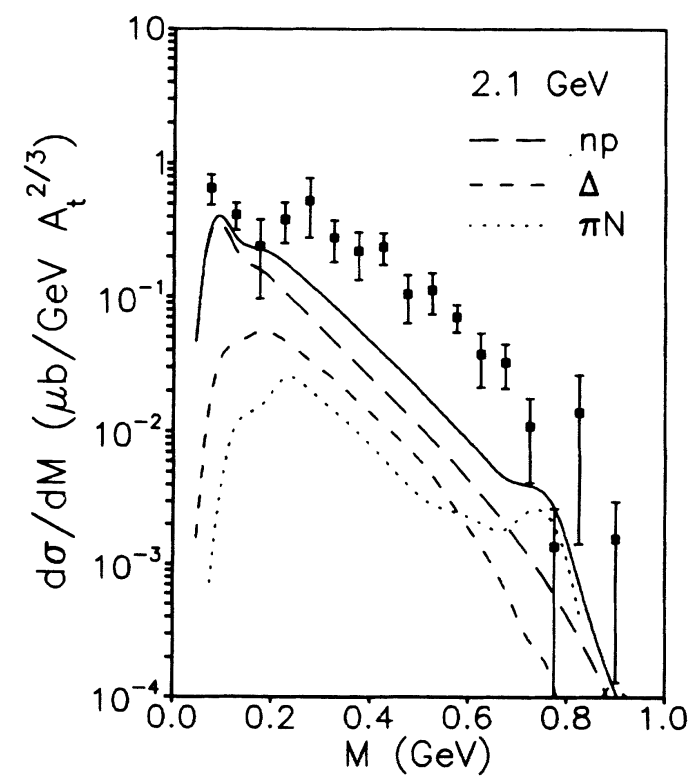

FIG. 4. Same as Fig. 3 for $p+$ Be reaction at $2.1 \mathrm{GeV}$.

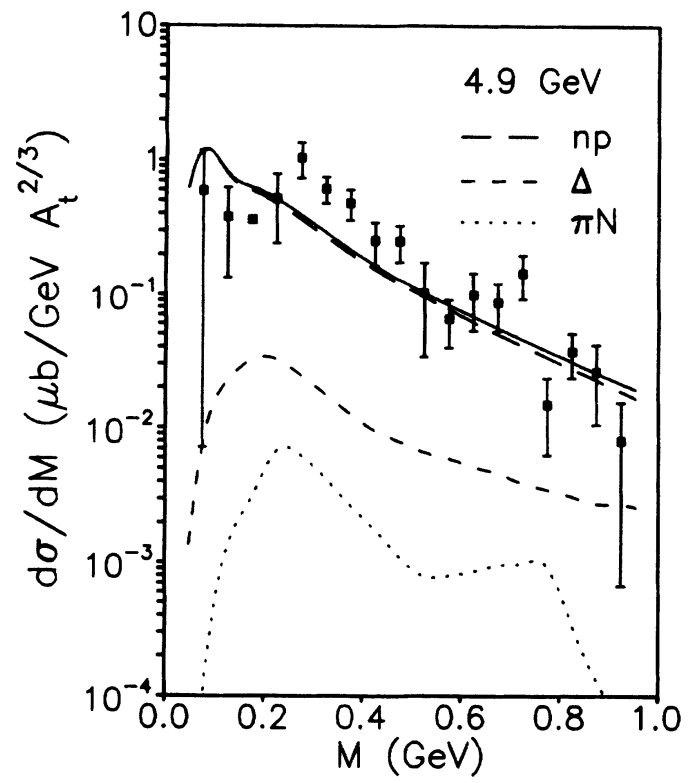

FIG. 5. Same as Fig. 3 for $p+$ Be reaction at $4.9 \mathrm{GeV}$.

teraction which also has negligible contribution except for dielectron pairs with invariant masses around the rho mass $m_{\rho}$ due to the rho-meson dominance of the pion electromagnetic form factor. It is noteworthy to point out that the contributions from both the delta decay and the pion-nucleon interaction will be larger if we do not introduce the form factors in evaluating the diagrams in Figs. 1 and 2. The small contribution from the delta decay and the pion-nucleon interaction at $4.9 \mathrm{GeV}$ explains why we still get reasonable agreement with the data even though our treatment of delta and pion production in the cascade

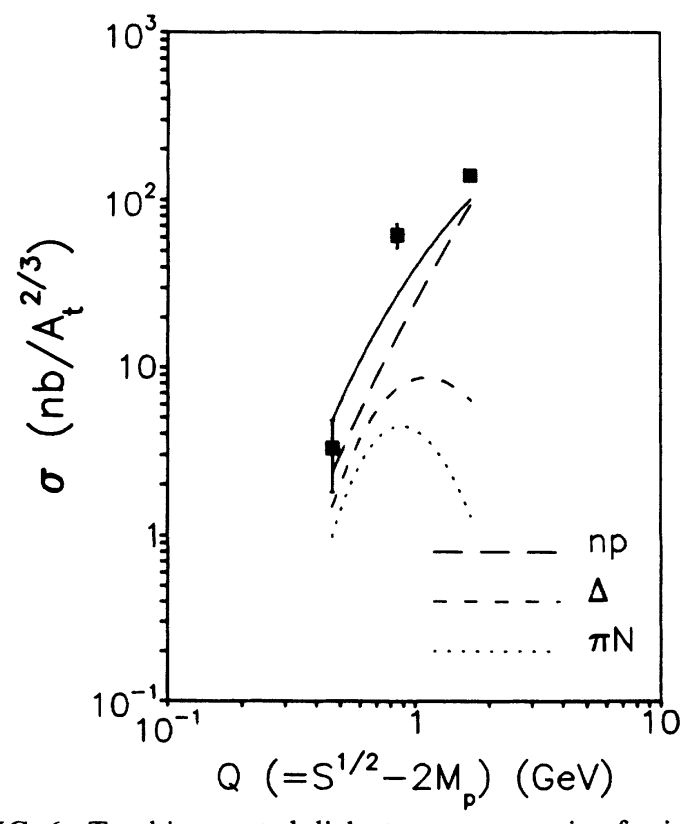

FIG. 6. Total integrated dielectron cross section for invariant masses $0.2 \mathrm{GeV} / c^{2}<M<0.7 \mathrm{GeV} / c^{2}$ as a function of the available nucleon-nucleon center-of-mass energy $Q$. 
model is crude at this energy. We note that about half the contribution from the proton-neutron bremsstrahlung is due to the first chance collisions. Our calculation does not show the peaks which appear at about $2 m_{\pi}$ and $m_{\rho}$ in the data. According to Ref. 9, the inclusion of pion-pion annihilation from multipion production in nucleon-nucleon collision can lead to the peak at $\boldsymbol{m}_{\rho}$. Whether the medium modification of the pion dispersion relation can explain the peak at $2 m_{\pi}$ as suggested in Refs. 3-5 needs further study.

In Fig. 6, we show the total integrated dielectron production cross section for invariant masses $0.2 \mathrm{GeV} / c^{2}$ $<M<0.7 \mathrm{GeV} / c^{2}$ as a function of the available nucleon-nucleon center-of-mass energy $Q$. The solid squares are the data from Refs. 1 and 2. Our theoretical results including all contributions are shown by the solid curve and are comparable to the data at $1.05 \mathrm{GeV}$ but are slightly smaller at 2 and $4.9 \mathrm{GeV}$. The contributions from the three different processes are also shown in Fig. 6. As expected, the contribution from the proton-neutron bremsstrahlung increases strongly with the energy of the incident proton. Because the delta production cross section from nucleon-nucleon interaction levels off at higher energies. Its contribution shows a similar energy depen- dence. The contribution from the pion-nucleon interaction, on the other hand, decreases at higher energies.

In summary, we have carried out a cascade calculation of dielectron production in proton-nucleus reactions. Including contributions from proton-neutron bremsstrahlungs, delta decays, and pion-nucleon interactions, we have obtained results which agree fairly with the measured data on $p+\mathrm{Be}$ reactions in view of the large experimental uncertainties. We find that dielectron production from the proton-neutron bremsstrahlung is the most important and requires thus careful study both experimentally and theoretically. Although both delta decay and the pion-nucleon interaction are unimportant for protonnucleus reactions, their contributions in nucleus-nucleus collisions remains to be investigated. ${ }^{15}$

We are grateful to $G$. Roche for sending us the program which simulates the detector acceptance filter. One of us (C.M.K.) would like to thank J. Kapusta, H. T. S. Lee, C. Naudet, and G. Roche for useful discussions. This work was supported in part by the National Science Foundation under Grants No. PHY-8608149 and No. PHY-8907986, and the Robert A. Welch Foundation Grant No. A-1110.
IG. Roche et al., Phys. Rev. Lett. 61, 1069 (1988).

${ }^{2}$ C. Naudet et al., Phys. Rev. Lett. 62, 2652 (1989).

${ }^{3}$ C. Gale and J. Kapusta, Phys. Rev. C 35, 2107 (1987).

${ }^{4}$ L. H. Xia, C. M. Ko, L. Xiong, and J. Q. Wu, Nucl. Phys. A485, 721 (1988).

${ }^{5}$ C. M. Ko, L. H. Xia, and P. J. Siemens, Phys. Lett. B 231, 16 (1989).

${ }^{6} \mathrm{M}$. Schäffer, T. S. Büro, W. Cassing, and U. Mosel, Phys. Lett. B 221, 1 (1989).

${ }^{7}$ K. Haglin, J. Kapusta, and C. Gale, Phys. Lett. B 224, 433 (1989).

${ }^{8}$ C. Gale and J. Kapusta, Phys. Rev. C 40, 2397 (1989).
${ }^{9}$ J. Kapusta and P. Lichard, Phys. Rev. C 40, R1574 (1989).

${ }^{10}$ J. Cugnon, D. Kinet, and J. Vandermeulen, Nucl. Phys. A379, 553 (1982).

${ }^{11}$ C. M. Ko and J. Aichelin, Phys. Rev. C 35, 1976 (1987).

12J. Aichelin and C. M. Ko, Phys. Rev. Lett. 55, 2661 (1985).

${ }^{13}$ Y. Kitazoe, M. Sano, H. Toki, and S. Nagamiya, Phys. Lett. B 166, 35 (1986).

${ }^{14}$ H. F. Jones and M. D. Scadron, Ann. Phys. (N.Y.) 81, 1 (1973).

${ }^{15}$ L. Xiong, Z. G. Wu, C. M. Ko, and J. Q. Wu, Nucl. Phys. A (to be published).

${ }^{16} \mathrm{H}$. Tanabe and K. Ohta, Phys. Rev. C 31, 1876 (1985). 